\title{
“Tecnologías creativas” Formación de habilidades y conocimientos relacionadas a las plataformas Scratch y Arduino en los niños y jóvenes de la comunidad urbano marginal del Distrito Metropolitano de Quito
}

\author{
Carlos Francisco Terneus Páez, Johanna Belén Tobar Quevedo, David Cesar Loza Matovelle, Fernando Naranjo Herrera \\ Departamento de Ciencia de la Energía y la Mecánica, Universidad de las Fuerzas Armadas - ESPE \\ cfterneus@espe.edu.ec, jbtobar@espe.edu.ec,dcloza@espe.edu.ec, lfnaranjo4@espe.edu.ec
}

\begin{abstract}
Resumen-La búsqueda incansable para hallar una vinculación entre la tecnología y la educación responde a la necesidad diaria del progreso y crecimiento económico y social de un país. Por esta razón el presente proyecto enlaza estos dos elementos fundamentales para la competitividad mundial, enmarcadas en un contexto de educación no formal posibilitando el acceso al aprendizaje de plataformas como son Scratch y Arduino, a partir de experiencias lúdicas las mismas que permiten un acercamiento a la ciencia de la programación. Dichos conocimientos fueron aplicados en proyectos ejecutados por niños y jóvenes de veinte "Casas Somos" ubicadas en el Distrito Metropolitano de Quito. Se obtuvo como resultado una potenciación del desarrollo cognitivo, razonamiento lógico, operaciones lógicas del pensamiento, ubicación espacial de los participantes, quienes propusieron como resultado la programación y diseño de diferentes proyectos enmarcados en la resolución de un problema de la vida cotidiana.
\end{abstract}

Palabras Claves- Arduino, Scratch, programación, niños, comunidades urbano marginales, lúdica, razonamiento lógicomatemático.

\begin{abstract}
The tireless search to find a link between technology and education responds to the daily need for progress, economic, and social growth of a country. For this reason, this project links these two fundamental elements for global competitiveness, ranged in a non-formal educational context, allowing access to learning platforms such as Scratch and Arduino, from recreational experiences, that allow an approach to the programming science. This knowledge was applied in projects executed by children and young people from twenty "Casas Somos" located in the Metropolitan District of Quito. It resulted in an enhancement of cognitive development, logical reasoning, logical operations of thought, spatial location of participants, who proposed as a result the programming and design of different projects ranged in the resolution of a problem of everyday life.
\end{abstract}

Keywords - Arduino, Scratch, programming, children, community, recreational activities, logical reasoning.

\section{INTRODUCCIÓN}

El proyecto nace de la necesidad de promover la inclusión económica, la promoción social y la supresión progresiva de la pobreza; enfocándose en zonas vulnerables con niños, niñas, adolescentes y jóvenes que son parte del programa de erradicación de trabajo infantil. En el año 2013 el Ministerio de Telecomunicaciones y de la Sociedad de la Información [3] aseguró que más del 60\% de las personas en el Ecuador tienen accesibilidad al internet; sin embargo, es imperante recalcar la escasa accesibilidad a recursos tecnológicos en zonas rurales de Quito.

Por tal razón, la Universidad de las Fuerzas Armadas en alianza estratégica ha desarrollado el proyecto de capacitación en programación orientado a dicho sector de la población; evidenciando el compromiso asumido en su campo de acción, y emprende las actividades necesarias para organizar los módulos y metodología de estudio. Obteniendo beneficios internos como: estudiantes universitarios participantes puedan desarrollar habilidades y competencias blandas en manejo de grupos, pedagogía, experiencia técnica y desenvolvimiento en público.

La aplicación se realizó en 20 "Casas Somos", pertenecientes a la ciudad de Quito. En ellas se disponen talleres artísticos, académicos y culturales destinados a niños, jóvenes, adultos y adultos mayores; dentro de los barrios y diferentes sectores de la ciudad [1], [2]. Se ejecutaron 23 talleres de capacitación con la temática en Scratch y Arduino, permitiendo el acceso al aprendizaje a 380 niños y 105 adolescentes y jóvenes.

El proceso de enseñanza-aprendizaje a niños y jóvenes para la programación está enfocado en plataformas amigables con el usuario, tales como: Arduino y Scratch. A través de las cuales es posible desarrollar capacidades cognitivas, entendiendo su importancia, ya que permiten la potencialización de habilidades perceptivas espaciales, lógica, procesamiento de información, recursos conceptuales, razonamiento, adicionalmente se inicia un acercamiento desde edades tempranas a la programación como ciencia y motivación al estudio de carreras técnicas [4], [5].

Dentro del artículo se presenta en la sección II se puede comprender el método utilizado para la ejecución del proyecto, fundamentación teórica en la que se exponen los programas utilizados y su relación con el desarrollo cognitivo que tienen los niños al programar. En la sección III se exponen los resultados más importantes encontrados tras la aplicación del proyecto, con el fin de comprender cuáles fueron los beneficios para los participantes en un sentido integral. Finalmente los resultados pueden ser observados en la sección IV. 


\section{MÉTODO}

La metodología utilizada fue de investigación-acción rural participativa, la cual pertenece a un campo cualitativo; no obstante, se tomaron en cuenta datos cuantitativos para medir el impacto de la misma. El proyecto reúne características, condiciones técnicas y operativas que los estudiantes han aprendido durante la trayectoria en las carreras de Mecánica y Mecatrónica. De manera complementaria se utilizaron estrategias lúdicas, las cuales permitieron aprendizajes significativos para los niños, quienes afianzaron sus conocimientos a través de la resolución autónoma de problemas [6]. Lo cual serviría de engranaje para lograr un enfoque multisectorial, el mismo que pretende fortalecer los vínculos de la Universidad de las Fuerzas Armadas-ESPE con sectores vulnerables durante todas las etapas del proyecto, las cuales se encuentran en la Fig. 1.

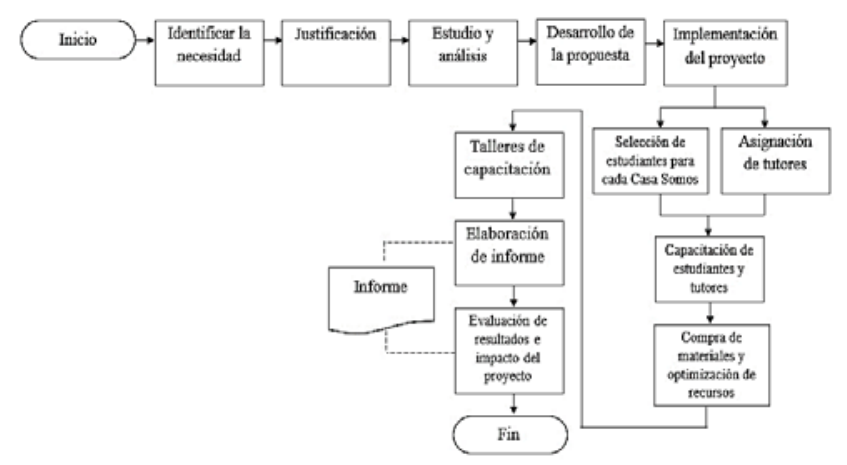

Fig. 1. Diagrama de flujo de etapas del proyecto.

\section{A. Desarrollo cognitivo en la infancia}

Mustard [7] asegura que: "La aplicación de este conocimiento (de desarrollo infantil) elevará la calidad de vida de la población, y favorecerá nuestro ideal de vivir en una sociedad más próspera, saludable, tolerante, pluralista, sostenible, democrática y pacífica”. Tomando en cuenta esta perspectiva se puede comprender la necesidad del trabajo mancomunado desde la educación y la tecnología para un desarrollo sostenible.

Estudios en los cuales demuestran que la edad infantil es la más importante y trascendente en la vida del ser humano, debido a la gran cantidad de neuronas cerebrales que realizan conexiones sinápticas, las cuales permiten aprendizajes significativos [8]. Por esta razón, la presente investigación se aplica en dicha población, con la finalidad de construir habilidades cognitivas para la resolución de problemas, gracias a la plasticidad cerebral, [9] que gracias al juego y experiencias individuales logran desarrollar conexiones neuronales, mejoran habilidades sociales, desarrollan operaciones mentales de orden superior, interacción intra e interpersonales [6].

\section{B. Operaciones lógicas del pensamiento}

A medida que se desarrolle el pensamiento lógico, se podrá construir conceptos abstractos que requieran razonamientos superiores, operaciones lógicas del pensamiento y por tanto desarrollar un pensamiento lógico que beneficie en sus decisiones cotidianas [10]:

- Clasificación: permite al niño conocer y desenvolverse en la realidad a través de agrupar las semejanzas y separar por diferencias con base a un criterio (o varios dependiendo de la edad).

- Seriación: consiste en establecer relaciones dentro de las diferencias y ordenarlas.

- Correspondencia término a término: a través de esta operación lógica el niño comprende que existe una relación uno a uno entre elementos agrupados en diferentes conjuntos para poder ser comparados de una manera cuantitativa.

\section{Beneficios de la programación a través de la lúdica}

Uno de los objetivos que busca la tecnología es mejorar la calidad de vida de las personas. Para ello, existe un proceso creativo que sigue una persona, no obstante estos elementos no responden a una continuidad cronológica ya que se deben considerar de manera integral dentro del mismo; se podría traducir como un caleidoscopio creativo hasta llegar a la invención [11].

De hecho, en la actualidad la humanidad vive rodeada de equipos que tienen funciones autónomas y colaboran con el ser humano, principalmente en la industria manufacturera realizando funciones repetitivas o peligrosas; pero también en servicios profesionales como inspección, rescate, limpieza; y también personales y domésticos como de seguridad en vivienda, para tareas dentro del hogar, entretenimiento, etc. Sin embargo, en el futuro cercano, se esperan grandes avances en los ámbitos de salud, la educación y domiciliares. La robótica en el futuro será una fuente de generación de empleos que serán originados por estas mismas tecnologías [12].

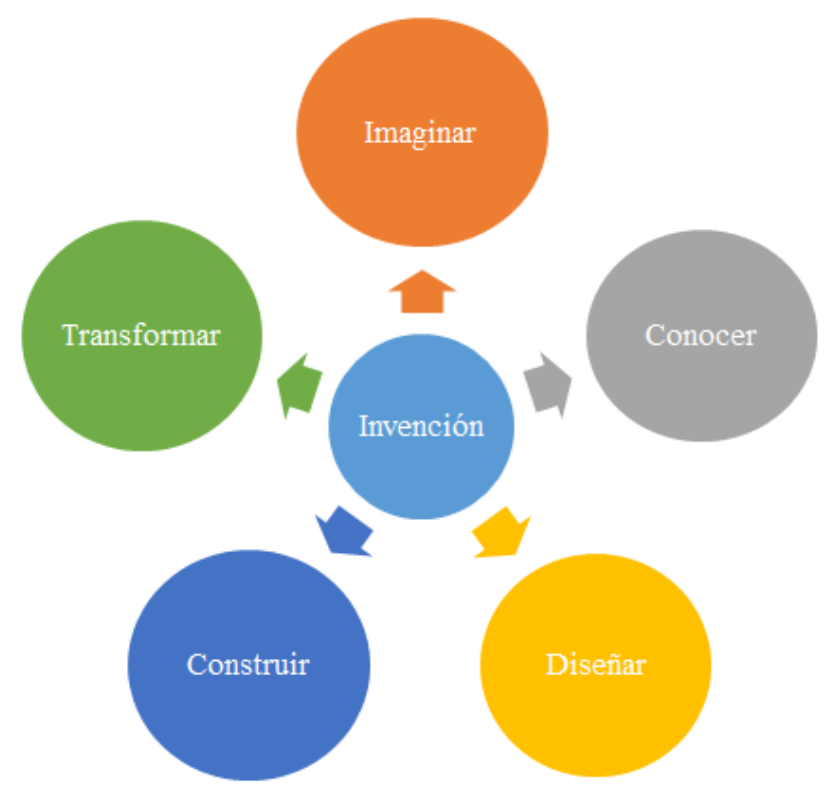

Fig. 2. Proceso creativo complejo de la invención.

\section{Plataformas accesibles}

Las plataformas utilizadas desde una perspectiva psicológica son efectivas porque logran mantener la atención focalizada del niño; además son motivadoras y estimulan la toma de decisiones y razonamiento [13]. El niño estructura sus ideas con lógica-matemática para dar órdenes de programación e ingresarlas en la plataforma.

Por otro lado, los participantes tuvieron la oportunidad de interactuar y experimentar con recursos electrónicos 
específicos logrando armar pequeños robots a quienes se les dio movimiento en base a condiciones de sensores. Esta interacción que mezcla la intuición con la teoría (verdades) son el sustento que [14] asegura que la experimentación habilita y mejora las condiciones de aprendizaje, debido a que los niños procuran actuar y construir de manera individual e independiente.

Adicional a ello, se puede resaltar el aporte de Ilvay [15] quien menciona el término de cibercultura y educación, en el cual se enmarca las caracaterísticas que requiere el software educativo, que son: ser flexibles y adaptables para diversos usuarios, que fomenten una comunicación interpersonal; y, que reciban retroalimentación. Es por esta razón que, se tomaron en cuenta la siguiente plataforma:

\section{E. S4A Scratch para Arduino}

Es una modificación de Scratch que permite programar la plataforma de hardware libre Arduino de forma sencilla, proporcionando una interfaz de alto nivel con funciones como la interacción con un conjunto de placas mediante eventos de usuario.

La interfaz presenta una organización por bloques, los objetos de Arduino para funcionalidades básicas del microcontrolador, escrituras y lecturas digitales y analógicas, permitiendo conectarse múltiples placas a la vez.

\section{ANÁLISIS DE RESULTADOS}

Al término del proyecto, se realizó la medición de impacto del proyecto a los coordinadores de las Casas Somos a través de entrevistas y encuestas, obteniendo los siguientes resultados:

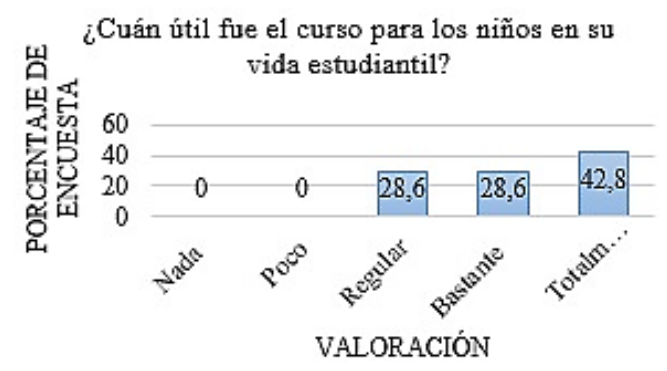

Fig. 3. Porcentaje de utilidad del curso

El $85,7 \%$ de la población encuestada consideró que la influencia del curso ha sido útil en el ámbito académico de los niños participantes. Teniendo en cuenta que para mejorar dicho ámbito es necesario desarrollar habilidades cognitivas específicas como las mencionadas anteriormente, se puede asegurar su influencia positiva dentro de la vida estudiantil. Por otro lado, se ha considerado a la concentración como una capacidad cognitiva básica para el desarrollo de las operaciones lógicas del pensamiento, y a través del análisis de resultados se pudo determinar que el $42,8 \%$ de los coordinadores considera que dicha capacidad ha aumentado totalmente en los niños participantes. Siendo un resultado representativo, ya que, a partir de la concentración surge la posibilidad de desarrollar, crear y plasmar nuevas ideas en los productos terminados, afianzando sus capacidades cognitivas.

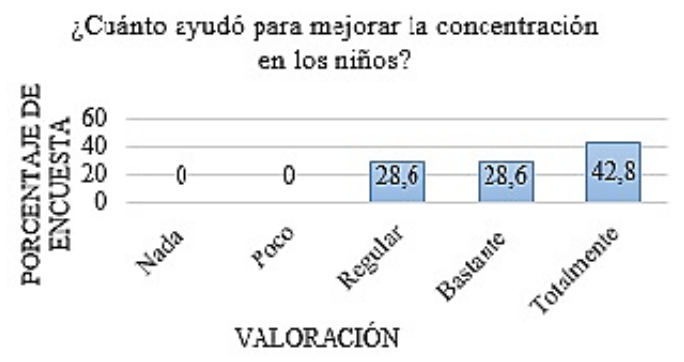

Fig. 4. Porcentaje de aumento de concentración

Se aplicaron encuestas para comprender desde una perspectiva personal la influencia de los cursos realizados, obteniendo que la mayoría de encuestados la consideró como positiva, debido a que, brindó oportunidades de crecimiento personal a comunidades rurales del país, recalcando que la metodología y teoría enseñadas los niños pudieron mejorar su destreza lógica matemática, solventar problemas, pensamiento creativo y acceso a información técnica. Estableciendo el impacto integral a la vida de los niños y jóvenes participantes del proyecto.

Adicional a ello, se aumentó en el niño el emprendimiento a través de actividades que potencien y orienten su vocación dirigida hacia una carrera tecnológica o de ingeniería, lo cual fue demostrado en las encuestas obteniendo que el $71,4 \%$ consideró que se aumentó el interés por dichas ramas de la ciencia. Por lo tanto, el integrar tecnología a la vida infantil en este tipo de talleres ayudó a desarrollar las habilidades mencionadas por Isbell \& Yoshizawa [20] en su libro "From Nuturing Creativity: An essensial mindset for young children's learning”, las cuales son: pensamiento creativo, flexibilidad, resolución de problemas y generar ideas innovadoras.

La introducción del conocimiento y apropiamiento de nuevas tecnologías desde edades iniciales, resultó beneficiosa en el desarrollo cognitivo desde varias perspectivas, una de ellas el pensamiento lógico-matemático y el fomento de habilidades para desenvolverse en la sociedad de la información. Evidenciado en los proyectos prácticos presentados por los niños participantes, quienes en la exposición final supieron exponer todo el procedimiento para su elaboración. Donde se pudo evidenciar el proceso creativo de los niños, al construir y diseñar la interfaz y jugar con las variables. Además, aprendieron a planificar, controlar el tiempo y tomar decisiones para lograr su objetivo. Es decir, se fortalecieron ya potencializaron las funciones ejecutivas, establecidas en el cerebro pre frontal [13].

Desde el campo tecnológico utilizaron conocimientos científicos y empíricos para solventar problemas técnicos y reales, optando por la solución que mejor se adaptó a las necesidades, costo, seguridad y otras condiciones del entorno, introduciéndose de esta manera en la solución de problemas para mejora la base de la producción del país.

Adicional a ello, este tipo de actividades permitieron potenciar la motivación, autonomía, desarrollo de distintas formas de comunicar sus ideas y mejorar sus habilidades sociales, reflexión, organización de ideas y trabajo en equipo; ya que tendrá la oportunidad de entrar en contacto con otros niños y compartir sus ideas y trabajos con otros, con el fin de buscar mejora a través de un trabajo 
mancomunado de descubrimiento y experimentación. [14]

El adolescente pudo desarrollar: dominio de responsabilidad sobre las circunstancias, combinar y movilizar sus saberes y actitudes, obtuvo nuevos aprendizajes de las situaciones a ser aplicados oportunamente, construcción y apropiación de nuevos recurso según la necesidad, canalizó el potencial creativo individual dentro de una acción colectiva.

\section{CONCLUSIONES}

Las herramientas de programación como Arduino y Scratch (S4A) resultan competentes para el acceso y enseñanza de programación y un acercamiento a la robótica gracias a su fácil acceso, bajo costo y presentación didáctica para ser utilizada por niños y jóvenes como un acercamiento a ciencias técnicas. Adicionalmente actividades de programación a través de la lúdica permiten un desarrollo integral en quienes las utilizan, gracias al desarrollo cognitivo que se logra fomentando el razonamiento, la reflexión, ubicación espacial, operaciones lógicas del pensamiento, análisis. Resulta necesario que la educación formal tome en cuenta estos procesos para lograr vínculos entre la tecnología y las nuevas generaciones, consiguiendo así nuevas propuestas.

\section{REFERENCIAS}

[1] La Hora, “Casa Somos ofrece más de 2300 cursos gratuitos,” La Hora, pp. https://lahora.com.ec/noticia/1102056405/casa-somosofrece-ms-de-2300-cursos-gratuitos-, 09 Mayo 2017.

[2] Municipio del Distrito Metropolitano de Quito, "Casas Somos. Municipio del Distrito Metropolitano de Quito,” 2018. [En línea]. Available: $\quad$ http://www.quito.gob.ec/index.php/municipio/66secretarias/277-casa-somos.

[3] Ministerio de Telecomunicaciones y de la Sociedad de la Información, "MINTEL y MIES promueven la inclusión de sectores vulnerables a la tecnología,” Ministerio de Telecomunicaciones y de la Sociedad de la Información, p. https://www.telecomunicaciones.gob.ec/?s=MINTEL+Y+MIES, 2013.

[4] K. A. Bonilla, Martínez y J. G. Rubio, Rodríguez, "Utilización del Software Scratch (S4A) y Hardware Arduino como mediadores en procesos educativos para promover el pensamiento algorítmico,” Bogotá, 2014.

[5] R. Blanco, Menéndez, "El pensamiento lógico desde la perspectiva de las neurociencias cognitivas,” Oviedo, 2009.

[6] B. E. Ojeda, Carpio, Tesis: Incidencia de la actividad lúdica como estrategia metodológica para el desarrollo del pensamiento lógico matemático en los niños y niñas del C.E.I. de "María Franco de Carrillo" y primer año de la escuela de educación básica "Galo Plaza Lasso"", Machala, 2015.

[7] J. F. Mustard , "Early Human Development - Equity from the Start Latin America," Revista Latinoamericana de Ciencias Sociales, Niñez y Juventud, pp. 639-680. Recuperado en: http://www.scielo.org.co/pdf/rlcs/v7n2/v7n2a04.pdf, 2009.

[8] A. Tinajero, "Trayectorias de neurodesarrollo en el ecuador: una lectura de los indicadores de desarrollo humano," http://docplayer.es/59289116-Trayectorias-de-neurodesarrollo-en-elecuador-una-lectura-de-los-indicadores-de-desarrollo-humanoalfredo-tinajero-febrero-2014-guayaquil.html, Guayaquil, 2014.

[9] M. R. Rosenzweig y E. L. Bennet, "Psychobiology of plasticity: effects of training and experience,” ELSEVIER, pp. 57-65, 1996.

[10] R. Rubio, Centeno, "El desarrollo lógico-matemático del niño a través de las tecnologías de la información y la comunicación,” Segovia, 2012.

[11] D. A. Hernández, Londoño, "Interfaz de programación visual como herrramienta educativo para el desarrollo de competencia en ciencia y tecnología por parte de niños, jóvenes y educadores,” Medellín, 2016.

[12] N. d. B. Jiménez, "Desarrollo de una plataforma didáctica para la educación infantil,” Madrid, 2015. .

[13] J. Tirapu, Ustárroz, J. M. Muñoz, Céspedes y C. Pelegrín, Valero, "Funciones ejecutivas: necesidad de una integración conceptual,"
Revista de Neurología, pp. 673-685. Recuperado en: https://pdfs.semanticscholar.org/f6f1/b7ec7671afea34cb9cfae7804ee1 3c369368.pdf, 2002.

[14] S. B. Hong y M. Trepanier-Street, "Technology: A Tool for Knowledge Construction in a Reggio Emilia Inspired Teacher Education Program,” Early Childhood Education Journal, pp. 87-94. Recuperado en: https://link.springer.com/article/10.1007/s10643-0047971-z, 2004.

[15] R. B. Ilvay, Taday, "Sistema de Educación para niños de 3 a 5 años, mediante un robot controlado por el sensor KINECT,” Riobamba, 2014.

[16] E. Arias, Méndez y G. Pereira, Carpio, “Enseñar Programación a niños, jóvenes y adultos usando la plataforma Arduino,” Simposio Argentino sobre Tecnología y Sociedad, pp. 44-50, 2016.

[17] P. Espeso, “Educación 3.0,” 27 Marzo 2017. [En línea]. Available: https://www.educaciontrespuntocero.com/experiencias/programacionrobotica-educacion-infantil/45272.html.

[18] J. Barrios, R. Kang, S. Morel, D. Kang, A. López y E. Martínez, "Inclusión Tecnológica de Niños mediante Robótica Educativa de bajo costo,” X Congreso de Tecnología en Educación \& Educación en Tecnología, pp. 624-630, 2015.

[19] L. M. Uribe, Sichaca y J. Vega, Africano, “A4C “Arduino For Childs: Una Alternativa Que Hace Uso De La Robótica Para El Desarrollo De Competencias Descriptivas,” Bogotá.

[20] R. Isbell y S. Yoshizawa, From Nurturing Creativity: An Essential Mindset for Young Children's Learning, The National Association for the Education of Young Children, 2016.

[21] E. Vara, Blanco, "La lógica matemática en Educación Infantil," Valladolid. 